\title{
An Unusual Granular Ulcer Secondary Oral Tuberculosis of Vestibule Mandibular Mucosa
}

\author{
Rocío Valenzuela-Narváez ${ }^{1}$ Daniel A. Valenzuela ${ }^{2}$ Daniel R. Valenzuela ${ }^{3}$ Aguilar Bailón ${ }^{4}$ \\ Heriberto Machco ${ }^{5}$ Carla Mena ${ }^{6}$ María Córdova ${ }^{2}$ Cris Mejía ${ }^{3}$ Ana Estrada ${ }^{4}$
}

${ }^{1}$ School of Dentistry, University of Barcelona, Barcelona, Spain

${ }^{2}$ National University Jose Faustino Sanchez Carrion, Huacho, Perú

${ }^{3}$ Military Hospital \& Gastrovital Medical Center, Perú

${ }^{4}$ Alas Peruanas University, Pueblo Libre, Perú

${ }^{5}$ National University Federico Villarreal, San Miguel, Perú

${ }^{6}$ San Martin de Porres Private University, Perú

\author{
Address for correspondence Rocío Valenzuela-Narváez, DDS, PhD, \\ School of Dentistry, University of Barcelona, Barcelona, Spain \\ (e-mail: violetvaln16@icloud.com).
}
Abstract
Keywords
- autograft
- buccal mucosa
- diagnosis
- granular ulcer
- secondary oral tuberculosis
- treatment

Tuberculosis (TB) is a chronic infectious disease caused by Mycobacterium tuberculosis. In the oral cavity, clinical manifestations are considered atypical lesions. The aim of this study was to report an unusual granular ulcer secondary oral TB that does not heal, chronic, had irregular appearance with deep depression of $2 \mathrm{~cm}$ in diameter, and was located in buccal mucosa of the premolar area-the left mandibular arch, of a 42-yearold woman. The patient was subjected to surgical excision of ulcer in its entirety and accordingly a periodontal plastic surgery in the area of the lesion was performed positioning an autograft subepithelial connective tissue. We obtained optimal results in improving oral health, function, and patient's comfort, in postoperative controls at 15,30 , and 60 days, respectively.

\section{Introduction}

Tuberculosis (TB) is a chronic infectious disease caused by Mycobacterium tuberculosis. Around eight million people are affected annually in the world and three million die every year due to complications of TB. ${ }^{1-4}$ TB is classified clinically as pulmonary and extra pulmonary. Pulmonary TB is the most common form of the disease while extra pulmonary TB ranges from 10 to $15 \%$ of infected people., 3

The lesions of oral TB are not common. Extrapulmonary TB develops mostly develop as chronic painless ulcers, between 0.05 and 5 cases. ${ }^{2,6}$ Primary oral TB is distinguished most often in young patients. The secondary oral TB frequently occurs in middle-aged and advanced adults. M. tuberculosis infects buccal mucosa, gingival mucosa, tongue, lingual frenum, and lips. It is most frequent in men compared to women. ${ }^{6-8}$

The aim of this study was to report a case of an unusual granular ulcer that did not heal, chronic, was of irregular appearance with deep depression of $2 \mathrm{~cm}$ in diameter, and was located in buccal mucosa of the area premolar the left mandibular arch of a 42-year-old woman with secondary oral TB.

\section{Case Report}

A 42-year-old female patient who attended a stomatological consultation for oral rehabilitation treatment underwent a detailed history. She was diagnosed with secondary oral TB. The patient received antituberculous therapy (ATT) with isoniazid INH, rifampicin (RMP), etambutol (EMB), and pyrazinamide (PZA), indicating improvement of her systemic condition but not of her oral clinical condition. At the intraoral clinical examination, the presence of a granular ulcer without cicatrization, chronic for 3 years behind the report of irregular edges, with deep depression of $2 \mathrm{~cm}$ in diameter and located in the vestibular mucosa of the premolar area of the left mandibular arch, was observed ( - Fig. 1). The pathological diagnosis confirmed an irregular surface of the ulcer covered with Trelat granules. Clinically asymptomatic with local lymph nodes are not enlarged. The anatomopathological study of the sample showed multiple stratified squamous epithelium with minimal caseous necrosis and Langhans giant cells ( $\mathbf{- F i g . 2}$ ). The patient was subjected to the clinical and surgical stomatological treatment protocol. The oral hygiene and the elimination of traumatic factors
License terms

(ㅇ)(1) $\Theta \circledast$ 


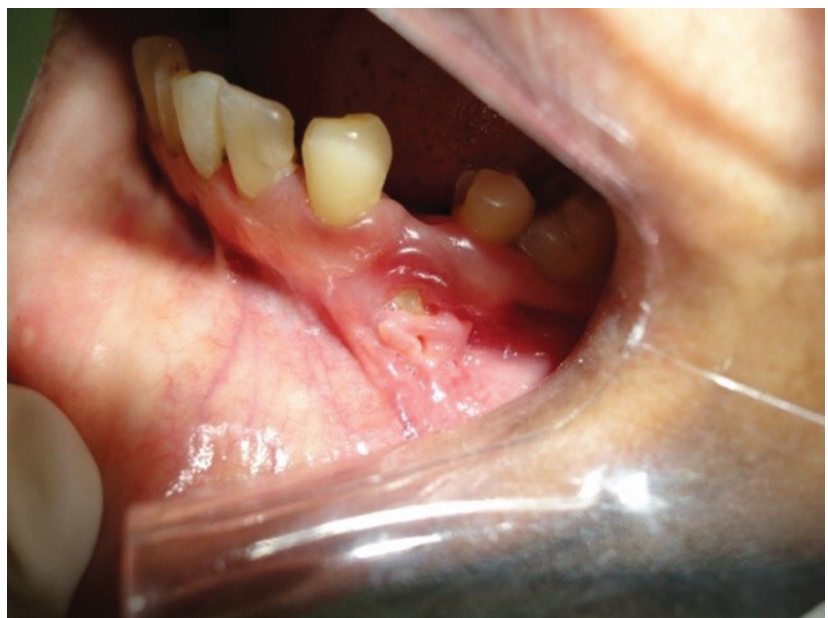

Fig. 1 The unusual granular ulcer that does not heal, chronic, of irregular appearance, with deep depression and located in buccal mucosa of the area premolar the left mandibular arch.

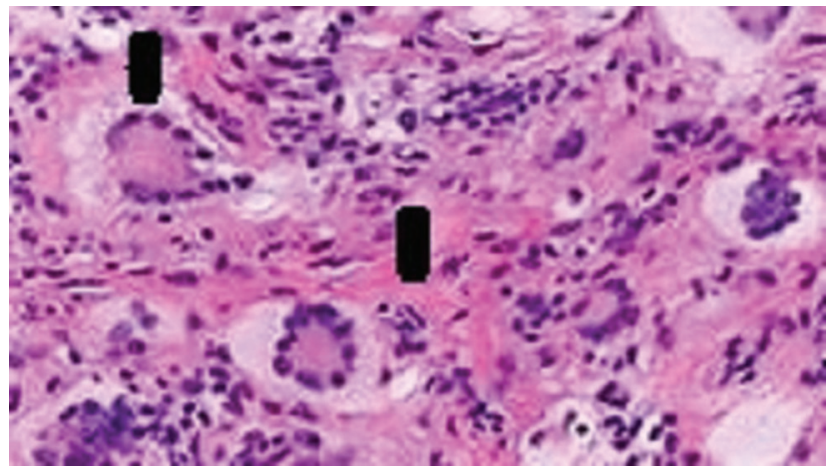

Fig. 2 The anatomopathological study of the sample showed multiple stratified squamous epithelium with minimal caseous necrosis and Langhans giant cells (black lines).

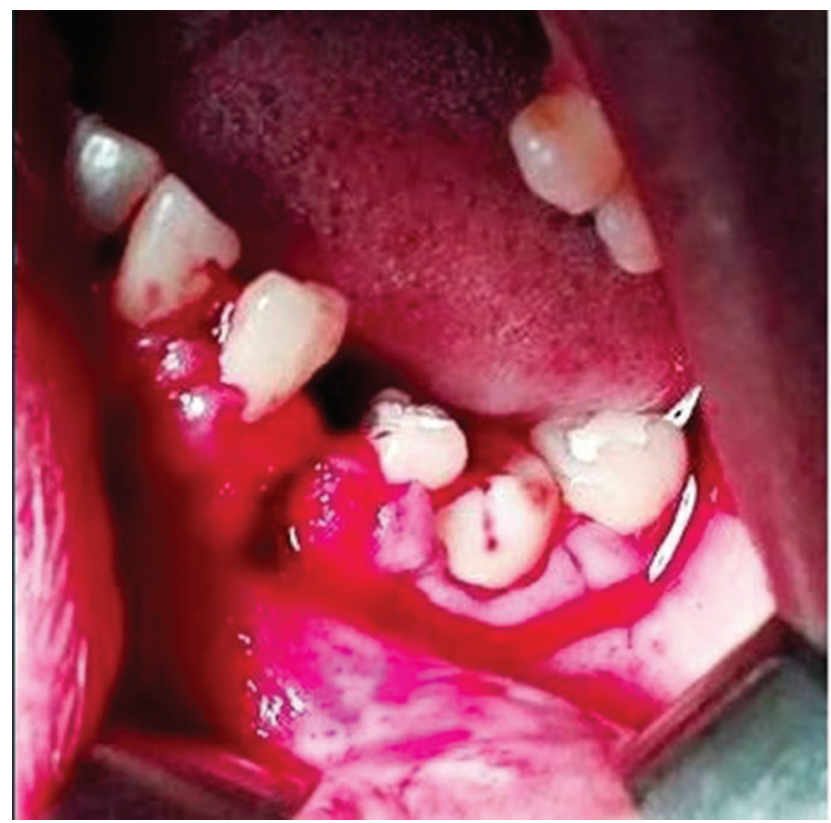

Fig. 3 Mucoperiosteal flap was performed with complete Neumann incision from 36 to 42, for the total excision of the granular ulcer secondary oral tuberculosis in all its extension projecting to $2 \mathrm{~mm}$ in healthy tissue adjacent to the area of the lesion. Check the edge of the flap is regular. The operative area was washed with sterile saline solution. were provided and re-evaluated before and after surgical treatment.

The inferior dental nerve and the buccal nerve were anesthetized with $2 \%$ lidocaine with epinephrine 1: 80,000. After anesthesia, a mucoperiosteal flap was performed with complete Neumann incision from 36 to 42, incising crestally in the edentulous and intrasulcular zone in the dentate area proceeding to the total excision of the granular ulcer secondary oral TB in all its extension projecting to $2 \mathrm{~mm}$ in healthy tissue adjacent to the area of the lesion ( - Fig. $\mathbf{3}$ ). Check that the edge of the flap is regular. The operative area was washed with sterile saline solution and consequently, periodontal plastic surgery was performed in the area of the lesion positioning a subepithelial connective autograft, which was extracted from the palatal area ( - Figs. 4 and 5). Erythromycin $500 \mathrm{mg} / 6 \mathrm{~h} / 10 \mathrm{~d}$ was prescribed along

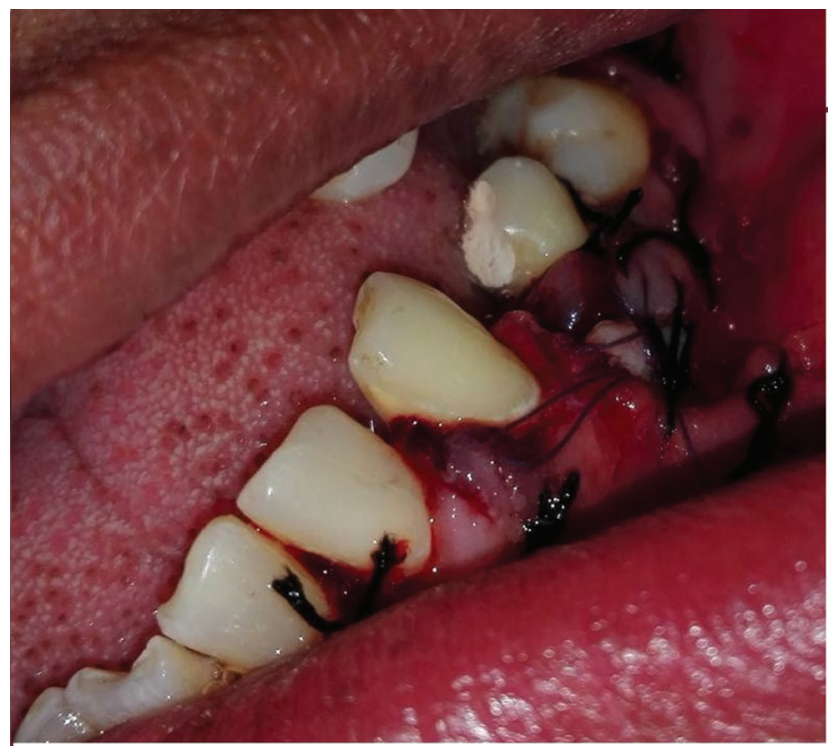

Fig. 4 Periodontal plastic surgery was performed in the area of the lesion. A sub epithelial connective autograft which was extracted from the palatal area and positionating in the area of the lesion.

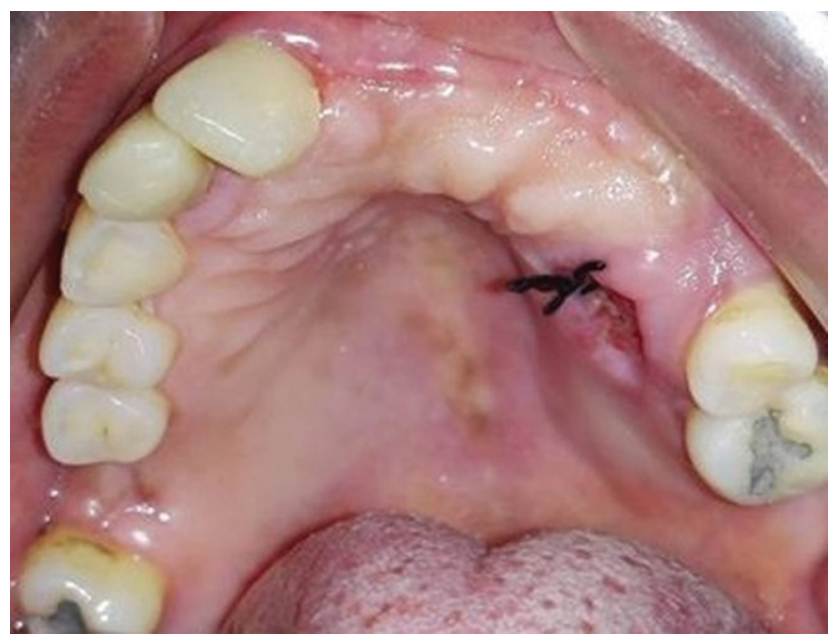

Fig. 5 Palatal donor area of the sub epithelial connective tissue with suture. 


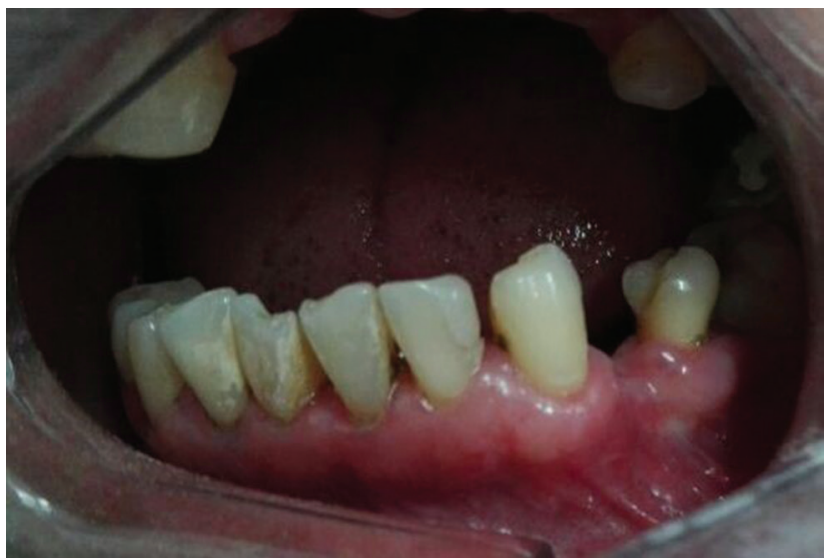

Fig. 6 Postoperative control at 30 days (front view).

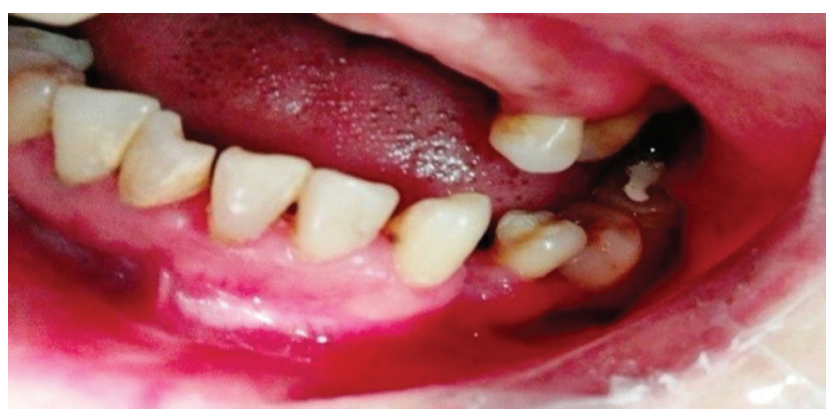

Fig. 7 Postoperative control at 60 days (side view).

with ibuprofen $400 \mathrm{mg} / 6 \mathrm{~h} / 3 \mathrm{~d}$, and oral rinsing with $0.12 \%$ chlorhexidine twice a day. After 10 days, the sutures were removed and the postoperative controls were performed at 15,30 , and 60 days, with the corrective treatment of the patient (-Figs. 6 and 7 ).

\section{Discussion}

Oral TB lesions are found only between 0.05 and 5\% of cases, developing in most of them as chronic painless ulcers. ${ }^{2-6}$ The pathological recognition of this entity is important and its early diagnosis is necessary. ${ }^{9-11}$ TB can infect all parts of the mouth such as soft and hard palate, uvula, buccal mucosa, gingiva, lips, tongue, maxilla, and jaw. ${ }^{6-11}$

According to published research by various authors two types of oral TB are recognized: primary oral TB which is more common in young patients and causes enlarged lymph nodes and secondary oral TB which is registered in adults of middle and advanced age with $M$. tuberculosis infecting buccal mucosa, gingival mucosa, tongue, lingual frenulum, and lips. ${ }^{12-14}$

We reported a routine examination in the oral cavity of a 42-year-old female patient, in whom a granular ulcer that does not heal was observed, chronic, of irregular appearance, with a deep depression of $2 \mathrm{~cm}$ in diameter, located in vestibular mucosa of the premolar area of the left mandibular arch was present. This finding of secondary TB according to the clinical manifestation did not present enlarged or painful local lymph nodes identifying an irregular surface ulcer covered with Trelat granules. The anatomopathological study of the sample showed multiple stratified squamous epithelium with minimal caseous necrosis and Langhans giant cells. Additionally, the clinical characteristics of the lesion aids in differentiating it from other pathologies in the oral mucosa such as syphilis, histoplamosis, planoepithelial cancer, or recurrent or aphthous stomatitis. ${ }^{9-11}$ Our finding is related to that reported by Krawiecka and Szponar et $\mathrm{al}^{14}$ and Erbaycu et al, ${ }^{15}$ who considered that the clinical characteristics of oral tuberculosis differ from other pathologies in the oral mucosa

According to Kakisi et al, the secondary form is observed more often than the primary one. ${ }^{6}$ The oral focus of infection, M. Tuberculosis, may appear as a result of autoinfection from the sputum with a route of hematogenous or lymphatic transmission. It is possible that in the majority of patients with TB, the constant flow of saliva and its antibacterial properties could protect the oral tissues from the invasion of the bacillus. However, it is also possible that local traumatisms in the oral cavity may promote infection, as referred by some researchers. ${ }^{6,14,15}$ We could also conclude, according to our study, that secondary oral TB in response to the invasion of the M. tuberculosis bacillus into the oral tissues is predisposed by local factors such as poor oral hygiene, prosthetic devices, and inadequate dental treatments.

We consider that the most important aspect of TB treatment is the ATT as a universal standardized drug therapy. In addition to this, we consider that after the opportune and differentiated diagnosis of the oral cavity in our case, we evidenced the necessity of the appropriate stomatological, clinical, and surgical treatment performing the total excision of the granular ulcer secondary oral TB and the periodontal plastic surgery. The ATT regimen consists of two phases: the firstline of treatment requires usually $\mathrm{INH}$, RMP, EMB, and PZA, which were administered for 2 months initially to continue in second phase for four consecutive months with INH and RMP. Inadequate management of TB or failure to apply the antibiotic regimen would produce resistance of the bacillus. A good follow-up of the regimen of adequate antituberculous treatment could prevent the oral TB. In this regard, we agree with what was reported by Pavlinac et al, ${ }^{1}$ Araj et al, ${ }^{2}$ Taute et al, ${ }^{3}$ Trinh et al, ${ }^{4}$ Nagaraj et al, ${ }^{5}$ and Aoun et al. ${ }^{12}$

However, when the lesion is established, it is necessary to consider the diagnosis of oral TB to determine the appropriate treatment for each patient. In relation to the surgical treatment of granular ulcer secondary oral $\mathrm{TB}$, the bibliographic evidence is scarce. However, local topical therapy with antiinflammatory ointments and oral mucosa protective agents are reported in investigations. ${ }^{6,14}$

In our case, in an intraoral clinical routine examination of the patient who underwent the detailed anamnesis and the clinical examination, it was possible to demonstrate the need to perform the corrective treatment. The total excision of the tuberculous granular ulcer was performed along with an immediate periodontal surgery in the area of the lesion positioning an autograft of subepithelial connective tissue. We agree with authors such as Chhina, ${ }^{16}$ Reino et $a l,{ }^{17}$ and 
Zuhr et al, ${ }^{18}$ on the use of the subepithelial connective graft for the treatment of plasty of large mucogingival defects.

We should mention that it was necessary to indicate to the patient about the improvement of her oral hygiene and the elimination of traumatic factors such as prosthetic devices and inadequate dental treatments.

The aim of this presentation was to report a case in a routine examination, of a granular ulcer that does not heal, of irregular appearance with a deep depression of $2 \mathrm{~cm}$ in diameter located in the vestibular mucosa of the premolar area of the arch, left mandible, of a 42-year-old female patient. Surgical excision of the tuberculous granuloma was performed in its entirety with a $2 \mathrm{~mm}$ projection to adjacent healthy tissue and consequently, periodontal plastic surgery in the area of the lesion positioning an autograft of subepithelial connective tissue extracted from the palatal area and the respective postoperative controls.

\section{Conclusion}

We considered it important to perform the detailed clinical examination of the patient with granular ulcer secondary oral TB, with the timely clinical, surgical, and stomatological treatment such as the surgical excision of the lesion and consequently, the periodontal plastic surgery with autograft of subepithelial connective tissue extracted from the palatal area. It reported optimal results in the improvement of oral health, function, and comfort of the patient treated at 15, 30, and 60 days, respectively. In all the time of study, we did not find recurrence of the pathology.

\section{Note}

The authors certify that they have contained all appropriate patient consent form. In the form, the patient gave her consent for the images and other clinical information to be reported in the journal. The patient understood that her name and initials will not be published and due efforts will be made to conceal her identify, but anonymity cannot be guaranteed.

\section{Funding \\ None.}

\section{Conflict of interest}

None declared.

\section{References}

1 Pavlinac PB, Lokken EM, Walson JL, Richardson BA, Crump JA, John-Stewart GC. Mycobacterium tuberculosis bacteremia in adults and children: a systematic review and meta-analysis. Int J Tuberc Lung Dis 2016;20(7):895-902

2 Araj GF, Saade A, Itani LY, Avedissian AZ. Tuberculosis burden in Lebannon: evolution and current status. J Med Liban 2016;64(1):1-7

3 Taute RB, Wylie J, Carter L. Oral and maxillofacial surgery: anunusual extranodal presentation of Mycobacterium Tuberculosis in the upper lip. Dent Update 2015;42(5):473-475

4 Trinh QM, Nguyen HL, Do TN, et al. Tuberculosis and HIV co-infection in Vietnam. Int J Infect Dis 2016;46:56-60

5 Nagaraj V, Sashykumar S, Viswanathan S, Kumar S. Multiple oral ulcers leading to diagnosis of pulmonary tuberculosis. Eur J Dent 2013;7(2):243-245

6 Kakisi OK, Kechagia AS, Kakisis IK, Rafailidis PI, Falagas ME. Tuberculosis of the oral cavity: a systematic review. Eur J Oral Sci 2010;118(2):103-109

7 Ferguson KA, McCormack DG. Tuberculosis involving the oral cavity. Can J Infect Dis 1993;4(1):12-14

8 Klepacz J, Peterson R, Kurnatowska AJ. Tuberculosis in oral cavity-a case report. Porad Stomatol 2008;8:169-172

9 Wang WC, Chen JY, Chen YK, Lin LM. Tuberculosis of the head and neck: a review of 20 cases. Oral Surg Oral Med Oral Pathol Oral Radiol Endod 2009;107(3):381-386

10 Ram H, Kumar S, Mehrotra S, Mohommad S. Tubercular ulcer: mimicking squamous cell carcinoma of buccal mucosa. J Maxillofac Oral Surg 2012;11(1):105-108

11 Gurfinkel PC, Gurfinkel AC, Cuzzi T, Ramos-e-Silva M. Eosinophilic ulcer of the oral mucosa. Skinmed 2012;10(4):228-231

12 Aoun N, El-Hajj G, El Toum S. Oral ulcer: an uncommon site in primary tuberculosis. Aust Dent J 2015;60(1):119-122

13 Rosado P, Fuente E, Gallego L, Calvo N. Primary tuberculosis of the palate. BMJ Case Rep 2014;2014:115-118

14 Krawiecka E, Szponar E. Tuberculosis of the oral cavity: an uncommon but still a live issue. Postepy Dermatol Alergol 2015;32(4):302-306

15 Erbaycu AE, Taymaz Z, Tuksavul F, Afrashi A, Güçlü SZ. What happens when oral tuberculosis is not treated? Monaldi Arch Chest Dis 2007;67(2):116-118

16 Chhina SA. 12 month clinical and radiographic study to assess efficacy of open flap debridement and subepithelial connective tissue graft in management of supra crestal defects. J Int Oral Health 2015;7:108-113

17 Reino DM, Maia LP, Novaes AB Jr, Souza SL. Comparative study of two surgical techniques for root coverage of large recessions in heavy smokers. Int J Esthet Dent 2015;10(3):456-467

18 Zuhr O, Bäumer D, Hürzeler M. The addition of soft tissue replacement grafts in plastic periodontal and implant surgery: critical elements in design and execution. J Clin Periodontol 2014;41(Suppl 15):S123-S142 